\title{
Desarrollo a escala humana y economía del bien común: estudio de caso en Fincomercio
}

\section{Human Scale Development and Economy of the Common Good: Fincomercio case study}

\author{
Paula Cristina Díaz Cruz \\ DOI: 10.29151/hojasyhablas.n18a4
}

\section{Resumen}

Las empresas desempeñan un papel importante en el desarrollo económico, dado que realizan actividades relacionadas con la producción de bienes y servicios, con el fin de satisfacer las necesidades de la población y aportar al crecimiento económico. Sin embargo, existen teorías alternas que identifican que dicho desarrollo no puede darse sin tener en cuenta el componente humano, mostrando cómo, a través de la identificación de las necesidades del entorno y la adopción de prácticas empresariales más profundas, se puede generar un desarrollo sostenible. Este artículo muestra el resultado de la aplicación de las metodologías de desarrollo a escala humana y economía del bien común dentro de la Cooperativa de Ahorro y Crédito (Fincomercio), donde se identifican las necesidades y los satisfactores de sus colaboradores como agentes creadores de valor dentro de la organización, y se evalúan sus contribuciones al bien común, mostrando algunas estrategias que favorecen el desarrollo, más allá de indicadores tradicionales.

Palabras claves: Escala humana; bien común; desarrollo; colaboradores; Fincomercio.
Companies play an important role in economic development, because they are carrying out activities related to the production of goods and services, in order to satisfy needs of the population, as well as to contribute to the economic growth. However, there are alternative theories that identify that such development cannot occur without taking into account the human component, showing how is possible to find the needs around the business area and to adopt deeper business practices to generate a sustainable development. This paper analyzes the result of human scale and economy of the common good methodologies that were applied by a credit cooperative (Fincomercio in Spanish), where the needs and satisfiers of employees, as value-creating agents of the organization, are identified and the contributions to the common good are evaluated, displaying strategies of development beyond traditional indicators.

Keywords: Human scale; common good; development; employees; Fincomercio.

\footnotetext{
${ }^{1}$ Economista. Magíster en Ciencias Económicas. Docente de la Fundación Universitaria Monserrate e integrante del Grupo de Investigación en Negocios y Mercados - GENEME. E-mail: pdiazc@unimonserrate.edu.co ORCID: 0000-0001-8411-4627
} 


\section{Introducción}

Actualmente, el desarrollo económico se entiende como el bienestar generalizado de una nación, el cual se logra a través de la aplicación de políticas públicas que están diseñadas para la salud, la educación, la alimentación, entre otros temas relacionados con el progreso. La efectividad de dichas políticas está dada por el crecimiento de los países, en sus principales indicadores económicos: Producto Interno Bruto (en adelante PIB), la reducción de la tasa de desempleo, el control de la inflación y la reducción de los índices de pobreza, buscando contribuir al bienestar y el mejoramiento de la calidad de vida de la población ${ }^{2}$.

Desde la Comisión Económica para América Latina y el Caribe (Cepal), en el área de desarrollo económico se tiene como objetivo la promoción de un crecimiento económico equitativo y de largo plazo a través de tres estrategias:

Un seguimiento sistemático de políticas y reformas macroeconómicas, una evaluación de la incidencia de éstas en términos de sustentabilidad, eficacia y equidad, y un apoyo sustantivo para la formulación e implementación de dichas políticas y reformas por parte de los gobiernos" (Cepal, 2019).

Es en este contexto que, las empresas aparecen como aquellas organizaciones donde se realizan actividades relacionadas con la producción de bienes y servicios, haciendo uso de los factores de producción -tierra, capital y trabajo-, los cuales satisfacen las necesidades de la población y contribuyen al crecimiento económico. Así, "el crecimiento en la productividad de un país es[tá] relacionado con el grado de innovación tecnológica y con el crecimiento capital y del trabajo en las economías por su participación en la producción" (López, 2013). Sin embargo, cuando se habla de crecimiento económico, se entiende en términos monetarios, pero no se contempla un crecimiento asociado al desarrollo social ni se evidencia una relación directa entre la riqueza de una nación para la reducción de la pobreza, haciendo cada día es más amplia la brecha entre ricos y pobres a nivel mundial,

Para el período 2017-2019 se prevén más problemas y un crecimiento insignificante del producto interno bruto (PIB) per cápita en África Central, Meridional y Occidental, Asia Occidental y América Latina y el Caribe. En el conjunto de esas regiones, 275 millones de personas viven en situación de pobreza extrema, lo que subraya la importancia de hacer frente a algunos de los problemas estructurales de más largo plazo que frenan el avance hacia el logro del desarrollo sostenible, así como de no dificultar la consecución de las metas de erradicar la pobreza $\mathrm{y}$ crear puestos de trabajo decente para todos. Si no se hace frente a esas cuestiones, un cuarto de la población de África podría vivir en situación de pobreza extrema en 2030 (Economic Analysis \& Policy Division, 2018).

Por esta razón, autores como Manfred MaxNeef (1986) y Cristian Felber (2010) proponen teorías alternativas, en las que las personas están en el centro del desarrollo. El desarrollo a escala humana y la economía del bien común hacen una profunda reflexión del sistema capitalista y cómo la actual economía de mercado ha generado una

\footnotetext{
${ }^{2}$ Es importante aclarar que existe una diferencia entre crecimiento económico y desarrollo económico. El primero hace referencia al aumento del valor en términos monetarios del Producto Interno Bruto de un país, en un periodo de tiempo determinado, es decir, el aumento de la riqueza; mientras el segundo hace referencia a cómo con esa creación de riqueza se mantiene el bienestar económico y social de los habitantes, más allá del indicador monetario. Martínez (2010) indica que el desarrollo económico es mucho más que el crecimiento o la caída de los ingresos de una nación, debido a que busca garantizar el ambiente necesario para que las personas y los grupos humanos puedan desarrollar sus potencialidades, y así llevar una vida creativa y productiva conforme con sus necesidades e intereses.
} 
serie de desequilibrios dentro de las diversas esferas que, han contribuido a la aparición de burbujas económicas, desigualdad, escases de recursos, crisis climáticas, hambrunas, crisis ambientales, crisis económicas, de índole social y político.

Las 26 personas más ricas del mundo concentran más del $50 \%$ de la riqueza mundial y el año pasado su fortuna aumentó en un $12 \%$, el equivalente a US\$ 2.500 millones diarios, mientras la de la mitad más pobre de la población mundial se redujo en un $11 \%$ (Weissman, 2019).

Manfred Max-Neef (1986) reconoció el fracaso de las teorías sobre el desarrollo, debido a que no tienen en cuenta el componente humano -las cualidades de los individuos-, afirmando que "el desarrollismo fue una experiencia profundamente movilizadora $[y]$ una experiencia generadora de ideas y de corrientes de pensamiento" (Max-Neef, Elizalde y Martín, 1986, p. 26) que no identificó de manera clara las necesidades de la población, las cuales son universales $y$, aquello que las hace distintas, son las formas en cómo se satisfacen.

De igual manera, "el desarrollo social y el desarrollo individual no pueden darse de manera divorciada. Tampoco es racionable pensar que el uno pueda sobrevenir mecánicamente como consecuencia del otro" (Max-Neef, Elizalde y Martín, 1986, p. 87), por ello es importante integrarlos a través de la matriz de necesidades y satisfactores, la cual reúne necesidades de carácter axiológico ${ }^{3}$ : subsistencia, protección, afecto, entendimiento, participación, ocio, creación, identidad y libertad; y existenciales 4 : ser (atributos personales), tener (instituciones, normas), hacer (acciones), estar (espacios y ambientes). Dicha relación es permanente y dinámica, lo que conlleva a una causación recíproca entre necesidades, satisfactores y bienes económicos, entendidos estos últimos como objetos y artefactos que afectan una necesidad de manera positiva o negativa. Así, partiendo de la matriz propuesta y de los satisfactores indicados, se da origen a diversas acciones que generan efectos sinérgicos para la satisfacción simultánea de las necesidades. Por ejemplo, la educación popular es considerada un satisfactor sinérgico, debido a que satisface directamente la necesidad de entendimiento, pero a su vez satisface las necesidades de protección, participación, creación, identidad y libertad.

De otro lado, Felber (2012) plantea una matriz que bien puede ser un instrumento que actúe como satisfactor de múltiples necesidades, es decir, un satisfactor sinérgico ${ }^{5}$; a esto se conoce como el enfoque de la economía del bien común, desde el cual se propone la integración del ser humano como indicador de medición dentro del sistema. Esta matriz comprende unos grupos de contacto, entre los cuales se encuentran proveedores, propietarios y proveedores financieros, personas empleadas, clientes y otras organizaciones, y el entorno social; así como unos valores que para el autor son universales: dignidad humana, solidaridad y justicia, sostenibilidad medio ambiental, transparencia y participación democrática. Para este autor, la dignidad humana es el mayor de los valores.

El objetivo propuesto para este artículo es identificar las necesidades y los satisfactores del recurso humano de la compañía Fincomercio, a través de la metodología de Manfred Max-Neef, integrada con la matriz de la economía del bien común de Christian Felber, estableciendo opor-

\footnotetext{
${ }^{3}$ Necesidades axiológicas: "Son aquellas que están relacionadas con el orden de los valores; es decir, con todas aquellas respuestas que tienden a satisfacer necesidades de cualquier índole: religiosa, artística, social, política, moral, etc" (Los hombres de ADE, 2006)

${ }^{4}$ Necesidades existenciales: Son aquellas necesidades que están asociadas a la persona y que son fundamentales para vivir como seres humanos.

${ }^{5}$ Satisfactores sinérgicos: Son aquellos que por la forma en que satisfacen una necesidad determinada, satisfacen simultáneamente otras necesidades.
} 
tunidades de mejora en las prácticas que tiene la Cooperativa, entidad que debido a sus características como empresa del sector solidario, busca contribuir al desarrollo y crecimiento del país, comenzando por el bienestar, desarrollo económico, humano, social y empresarial de sus colaboradores y asociados. La pregunta de investigación que se plantea responder es ¿Cómo Fincomercio contribuye a mejorar el desarrollo social y económico, por medio de la identificación de los satisfactores a escala humana, dentro de la organización y su integración con la matriz de la economía del bien común?

El desarrollo humano es algo que le compete a la sociedad actual. Dentro del capitalismo no se tiene en cuenta este factor de trabajo como un elemento decisivo dentro de los procesos de producción de bienes y la generación de servicios, haciendo ver que el ser humano está diseñado para el sistema cuando debería ser lo contrario. Por ello, es importante identificar las necesidades de los colaboradores dentro de las organizaciones, al evaluar cómo las mismas contribuyen al bien común, junto con sus satisfactores, visibilizando las estrategias que favorezcan un desarrollo más allá de los indicadores tradicionales.

\section{Metodología}

El método empleado fue inductivo, iniciando con la identificación de las necesidades por parte de los colaboradores de la empresa, quienes fueron seleccionados de manera aleatoria y pertenecen a distintas áreas de la organización: comerciales, operativos, gestión humana, entre otros. De igual manera, se aplicaron instrumentos cualitativos, principalmente desde las metodologías de las matrices propuestas por Max-Neef y Felber, las cuales se detallan a continuación:

A partir del enfoque de desarrollo a escala humana, se realizó un taller divido en 6 etapas:

Primera etapa: Se toma un número de 50 personas, teniendo en cuenta que es un número óptimo para alcanzar los objetivos planteados dentro del desarrollo del taller. Así, la muestra se divide en 5 grupos de diez personas; para ello se propone a cada grupo elaborar la matriz que contenga los satisfactores negativos, es decir, los que afectan su entorno laboral (a nivel macro se habla de su sociedad), que impiden la actualización de las necesidades humanas fundamentales (MaxNeef, Elizalde y Martín, 1986). Para el caso del ejercicio en Fincomercio, se diseñaron 5 matrices en blanco y se pasaron papeles de colores por cada una de las categorías existenciales.

Segunda etapa: Se pide al grupo diligenciar los casilleros correspondientes a la columna ser. Cada satisfactor que se ingresa es producto de la discusión de grupo, y en esta columna se deben registrar atributos personales o colectivos. Luego se diligencia la columna tener, en esta se registran instituciones, normas, mecanismos, políticas etc. Se establece una pausa y se retoma para diligenciar las columnas hacer y estar; en la primera se registran acciones personales o colectivas y se expresan como verbos, tales como imponer, censurar, adoctrinar, manipular, entre otros. En la segunda se registran espacios o entornos pues se hace referencia a tiempos y lugares, por ejemplo, oficina, puestos de trabajo, espacios abiertos, etc.

Tercera etapa: Después del ejercicio, los talleristas hacen una depuración de la matriz, buscando sinónimos en cada una de las categorías para unificar las matrices.

Cuarta etapa: En un segundo ejercicio se muestra a los participantes la matriz depurada y mediante una discusión de grupo se escoge el satisfactor o común denominador más importante y decisivo en cada una de las categorías, en casos excepcionales, se puede hablar de dos satisfactores.

Quinta etapa: Se presenta la matriz síntesis y resultado de la discusión de la etapa anterior. Esta matriz representa los desafíos principales 
que deben abordarse dentro de la organización. Es importante que dicha matriz sea producto de la discusión del grupo.

Es importante aclarar que, en esta investigación se hizo una adaptación de la metodología propuesta para la Cooperativa Fincomercio, teniendo en cuenta la escasez de tiempo disponible para su aplicación. De este modo, el ejercicio de participación se realizó en menor tiempo del establecido y sólo se hizo énfasis en términos negativos, es decir, hasta la tercera etapa. Para Max-Neef, la matriz no es normativa, por lo tanto, el moderador de la actividad puede hacer una clasificación de los satisfactores, de acuerdo a la experiencia lograda con el grupo.

Desde el enfoque de la economía del bien común, se habla de un balance que mide "cómo los puntos centrales consensuados que lo componen son experimentados por la empresa" (Felber, 2012 , p. 53) haciendo una evaluación de la interacción que existe entre los grupos de interés de la organización y los valores fundamentales. Se evalúan 20 temas entre los cuales se encuentran las condiciones laborales, la utilidad de los productos o servicios, las remuneraciones de los trabajadores, el reparto de los ingresos, la relación de las empresas con proveedores, clientes y accionistas, entre otros.

Estos temas se califican con valores establecidos entre 0 y 10 , para ello existe una herramienta diseñada por parte de la organización del bien común sobre la cual, el autor del balance puede trabajar y evaluar dichos temas. "La valoración se realiza teniendo en cuenta los niveles de aportación al Bien Común (punto de partida, primeros pasos, avanzado, experimentado y ejemplar). Para este fin, debe utilizarse toda la información e indicadores disponibles en la Matriz EBC" (Equipo de redactores, 2017, p. 9), haciendo énfasis en los temas evaluados dentro de la organización.

Si bien algunas compañías utilizan mediciones de sus procesos financieros y productivos desde la responsabilidad social corporativa, no se presentan análisis de manera detallada, como lo proponen Max-Neef y Felber. Por tanto, lo que pretende esta investigación es integrar ambas metodologías ${ }^{6}$, generando un efecto sinérgico que le sirva a la organización dentro de sus procesos internos y externos. El supuesto es que se dará un impacto positivo frente al desarrollo empresarial, entendido como el progreso de las empresas en términos de competitividad y productividad responsables socialmente y desde un ambiente laboral de bienestar.

\section{Resultados y Discusión}

Las sistematizaciones propuestas en las metodologías utilizadas en esta investigación son aplicables para fines de diagnóstico, planificación y evaluación. Para este estudio de caso, se obtuvieron los siguientes resultados:

\section{Diagnóstico}

Fincomercio es una cooperativa de ahorro y crédito fundada el 26 de noviembre del año 1957. Durante los primeros 35 años funcionó como fondo de empleados del Banco de Comercio, y a partir del año 1992 empezó a funcionar como cooperativa. Su objetivo principal es proporcionar inclusión financiera a sus más de 190.000 asociados y 840 empresas, creando una rentabilidad social "a través de servicios financieros de fácil acceso para empleados, independientes, pensio-

\footnotetext{
${ }^{6} \mathrm{Al}$ integrar las teorías de Max-Neef y Felber se busca complementariedad, debido a que presentan necesidades, valores y grupos de interés que tienen una característica particular: la universalidad. Así, al identificar estos elementos dentro de las organizaciones, en el caso del desarrollo a escala humana, los satisfactores de las necesidades desde el ser, el tener, el hacer y el estar; junto con la matriz del bien común, se puede ir más allá de un proceso productivo tradicional, haciéndolo más humano.
} 
nados, universitarios y taxistas" (Fincomercio, 2019). La Cooperativa ofrece servicios de ahorro e instrumentos financieros como créditos, seguros generales y convenios con otras entidades de recreación. Con cerca de 400 colaboradores, entre los que se encuentran personal de planta, temporales, aprendices del Sena y corretajes, se ha preocupado por ser una cooperativa que busca generar un impacto positivo dentro y fuera de su organización.

Construcción de una matriz de necesidades organizacional

Entender el desarrollo empresarial dentro de una organización implica que el empresario tenga en cuenta una serie de elementos como el crecimiento económico, la cultura empresarial, el liderazgo, la gestión del conocimiento e innovación. Sin duda, uno de los elementos más importantes es el componente humano, pues es un concepto integrador con el que se puede lograr un impacto positivo en las organizaciones, mediante el reconocimiento de las capacidades del capital humano (Delfin y María, 2016). A continuación, se muestra la matriz obtenida del ejercicio realizado con colaboradores de Fincomercio en mayo de 2019:

\section{Tabla 1 Matriz negativa sintetizada}

\begin{tabular}{|c|c|c|c|c|}
\hline \multirow{2}{*}{$\begin{array}{l}\text { Categorias } \\
\text { Axiológicas }\end{array}$} & \multicolumn{4}{|c|}{ Categorias existenciales } \\
\hline & SER & TENER & HACER & ESTAR \\
\hline \multirow{2}{*}{ SUBSISTENCIA } & Estrés & Transporte & Relajarse & Entomo social \\
\hline & & Horarios extensos & & \\
\hline \multirow[t]{2}{*}{ PROTECCION } & Elitismo & Ingreso & $\begin{array}{c}\text { No trabajar en } \\
\text { equipo }\end{array}$ & Entomo social \\
\hline & & & & Puesto de trabajo \\
\hline AFECTO & Intolerancia & Brecha generacional & Tolerar & $\begin{array}{c}\text { Espacios de } \\
\text { integración }\end{array}$ \\
\hline ENTENDIMIENTO & $\begin{array}{c}\text { Desconfianz } \\
\text { a } \\
\end{array}$ & $\begin{array}{c}\text { Herramientas } \\
\text { insuficientes } \\
\end{array}$ & Devaluar & $\begin{array}{l}\text { Acceso a la } \\
\text { informacion } \\
\end{array}$ \\
\hline \multirow{2}{*}{ PARTICIPACION } & $\begin{array}{c}\text { Autoritarism } \\
0 \\
\end{array}$ & $\begin{array}{c}\text { Desigualdad en los } \\
\text { cargos }\end{array}$ & Imponer & Centralización \\
\hline & $\begin{array}{c}\text { Conformism } \\
0\end{array}$ & & & \\
\hline OCIO & $\begin{array}{c}\text { Despreocupa } \\
\text { cion }\end{array}$ & Congestion & Planificacion tareas & $\begin{array}{c}\text { Ambientes para } \\
\text { descanso }\end{array}$ \\
\hline \multirow{2}{*}{ CREACION } & Limitacion & Exceso de trabajo & Desvalorizar & $\begin{array}{l}\text { Espacios } \\
\text { monótonos }\end{array}$ \\
\hline & $\begin{array}{c}\text { Adicción al } \\
\text { trabajo }\end{array}$ & & & \\
\hline IDENTIDAD & Inseguridad & Desigualdad & Aislar & Integraciones \\
\hline LIBERTAD & Sumision & Prejuicios & Manipular & Entomo social \\
\hline
\end{tabular}

Fuente: Elaboración propia con base en el ejercicio metodológico propuesto 
Interpretación de la matriz de necesidades de Fincomercio

Dentro del contexto empresarial, existen una serie de factores o elementos negativos que afectan a colaboradores en el desarrollo de sus actividades y pueden llegar a limitar su productividad, sus relaciones e interacción con el entorno laboral y su equipo de trabajo. El análisis realizado para los satisfactores resultantes de la aplicación de la metodología, constituyen una herramienta que permitirá establecer estrategias, modelos y programas que Fincomercio puede implementar para alcanzar una mejora sustancial del desarrollo de su capital humano, logrando a la vez el crecimiento y desarrollo de la Cooperativa, sus asociados y el público interesado.

El motivo por el cual se decidió hacer la investigación en Fincomercio se relaciona con la experiencia laboral que la investigadora tuvo dentro de la organización, de la cual hizo parte durante 4 años. A lo largo de este tiempo, se relacionó con un ambiente laboral agradable, desde sus directivos, empleados y demás miembros, percibiendo compromiso y calidad humana; así, se propone a la Cooperativa desarrollar el proyecto de investigación desde la aplicación de las teorías propuestas, debido a que ambas no se han aplicado de manera conjunta en alguna organización colombiana, y debido a la actividad económica que desempeña es un potencial para generar propuestas de mejora a sus actividades a nivel organizacional y social.

El grupo de 50 trabajadores son personas cuyas edades oscilan entre los 20 y 60 años, quienes se seleccionaron de manera aleatoria, $y$ hacen parte de diferentes gerencias de la compañía: comercial, operativa y talento humano. La aplicación de la metodología permitió identificar aspectos relevantes que se narran a continuación.

Subsistencia. Entendida como todos aquellos medios que permiten a las personas mantenerse vivas. Para los colaboradores de Fincom- ercio, desde la parte del ser, esta definición se ve afectada por el estrés laboral, uno de los mayores causantes de enfermedades psicológicas y físicas que afectan la eficacia de las personas (OMS, 2019). La subsistencia es una categoría axiológica que define las necesidades más básicas del ser humano, como lo son su propia existencia y su instinto de supervivencia. Dicha categoría encabeza la matriz, permitiendo establecer como prioridad los satisfactores asociados al ser, tener, hacer y estar, como el punto de partida de este análisis e identificando el manejo del estrés como uno de los satisfactores que más requiere de atención. Por ejemplo, factores como el transporte al sitio de trabajo, los horarios extensos y los ambientes de trabajo inadecuados influyen en el estado de ánimo, la productividad y eficiencia de los colaboradores; siendo necesario contar con espacios para relajarse en el ambiente de trabajo.

Protección. Es la necesidad que tienen las personas de sentirse protegidas y seguras. La protección como categoría básica es a la vez trascendente dentro del ser humano y se extiende desde el individuo en sí, hacia los círculos de su entorno social, desde el más cercano como su familia, hasta los más lejanos como su comunidad o su país. Además, es una necesidad con equilibrio frágil, $\mathrm{y}$ desde la perspectiva de un trabajador, aspectos como el acceso a la seguridad social, la asignación salarial o la posición frente a su entorno socio laboral afectan su satisfacción. Para los colaboradores de Fincomercio, el ingreso y el estatus dentro de la organización afectan su necesidad de protección, dado que dificultan el relacionamiento entre pares, el trabajo en equipo y el entorno en general, siendo necesario desarrollar, estimular satisfactores que disipen las diferencias que la generan.

Un estudio de empleo realizado en Reino Unido en el año 2015, mostró que para trabajar en empresas selectas británicas el 70\% de los empleados de dichas compañías procede de escuelas privadas y bloquean a personas de clase obrera (Gimón, 2015) debido al estilo personal, el acento 
y las buenas costumbres. Estos son algunos aspectos que se evalúan a la hora de ocupar altos cargos, limitando a aquellas personas que no tienen ciertos privilegios como escuelas de formación prestigiosas, lo cual cierra la posibilidad de acenso social.

De alguna manera, lo anterior influye en el ingreso de las personas asociado al nivel de formación y capacitación de los colaboradores, generando que muchos no logren ascender en sus puestos de trabajo y mejorar sus ingresos dentro de la compañía. Por ello, se deben tener en cuenta aspectos más allá del nivel de formación, tales como la experiencia en el cargo, el conocimiento dentro de la compañía, habilidades blandas, los años laborados, entre otros, como elementos a tener en cuenta a la hora de promover a un colaborador.

Afecto. Se diría que, en términos empresariales, el afecto como categoría axiológica no tendría lugar; sin embargo, es importante no dejar de lado que el ser humano evoluciona constantemente a través de su relación con el mundo, y con los demás seres humanos, y su desarrollo afectivo se extiende hacia todas sus interacciones, siendo fuente constante de destructores o satisfactores de carácter social e individual. En el ámbito laboral, el prolongado tiempo de interacción entre colaboradores hace que la generación de satisfactores asociados al afecto sea abundante y significativa, convirtiendo elementos como el reconocimiento, la tolerancia, la aceptación, la semejanza y la diferencia en insumos que alimentan de manera constante el nivel de satisfacción o insatisfacción del colaborador, y a la vez su relación con su lugar de trabajo y el entorno de la compañía.

En Fincomercio, de acuerdo con la síntesis obtenida durante el ejercicio, se evidencia que la intolerancia y la brecha generacional son los aspectos que hacen del ambiente laboral un asunto difícil de sobrellevar, generando entre los colaboradores incomodidad con su labor diaria, siendo necesaria la generación de espacios de integración con el fin de mejorar los términos de tolerancia y la interrelación de cada uno de los colaboradores.

Así mismo, la brecha generacional puede representar una oportunidad o un obstáculo en la consecución y éxito en los resultados de las empresas. Algunas situaciones de conflicto que se presentan dentro de las compañías están dadas por la impaciencia, el narcicismo, la pereza, lo anticuado, la rigidez de pensamiento, etc.; así "al estar motivados por expectativas e intereses distintos, no es fácil que jóvenes, adultos y veteranos formen equipos productivos, pero la clave para que alcancen el máximo desempeño es lograr que cada individuo entienda la lógica del comportamiento de los demás" (Solo consultores en cambio, 2015). La importancia de la convivencia generacional entre colaboradores fortalece las relaciones entre ellos mismos; por tal razón, es importante establecer metas comunes y aclarar que el éxito de la organización está dado por el trabajo en equipo, sin importar sea cuál sea la edad y la profesión de la persona.

Entendimiento. En el ambiente laboral, el entendimiento tiene un marcado efecto como necesidad, por cuanto es la oportunidad para que cada ser humano muestre sus actitudes y aptitudes, sus conocimientos, sus habilidades y capacidades en acción. Al igual que el afecto, los satisfactores asociados a esta necesidad son alimentados constantemente por sentimientos de reconocimiento, competitividad, inexperiencia, desconfianza, aprobación o devaluación.

Por otra parte, el constante flujo de información y la tecnología constituyen factores que facilitan o dificultan que un colaborador se relacione armónicamente con su puesto de trabajo. En Fincomercio, algunos de ellos sienten que la desconfianza en sus conocimientos, la devaluación de las capacidades, la dificultad de acceso a la información y la falta de herramientas tecnológicas son factores que marcan de manera significativa 
las condiciones de trabajo en la Cooperativa.

Según Pereira (2011), un proceso que puede implementar la Cooperativa para mejorar este tipo de situaciones esta dado en lo que se denomina "implementación del conocimiento en la empresa", partiendo desde la identificación del conocimiento, la retención y utilización del mismo, la manera en cómo se distribuye dentro de la organización, como se desarrolla y finalmente cómo se adquiere por parte de los colaboradores.

El éxito de este procedimiento está dado en la capacidad que tienen los líderes en valorar los conocimientos de sus colaboradores, formando equipos que mantengan informados a los que no participen del proceso y siendo capaces de trasmitirlo de manera efectiva para potenciar las habilidades de la organización.

Participación. Aunque en un ambiente laboral, la estructura organizacional lleve a pensar que los canales y alcances de la participación estén definidos, en el caso de muchas organizaciones este se puede ver entorpecido por temas relacionados con el autoritarismo, la desigualdad en los cargos y el conformismo por parte de los colaboradores, tal como se muestra en la matriz desarrollada por el grupo.

Es así como, generar espacios donde se cambie la mentalidad de los colaboradores, a través de la innovación, es un reto que hoy en día todas las organizaciones deben asumir. La innovación "exige que las mediocridades y los conformismos en el trabajo desaparezcan y que cada uno de los trabajadores se muestran dispuestos a arriesgar y emprender a través de las ideas y los grandes cambios" (Pérez, 2018 citada por Americaeconomía. com, 2018). Entonces, es importante hacer que los colaboradores participen cuando se quiera lanzar un nuevo producto o mejorar un proceso, pues esto hace parte de un mejoramiento continuo dentro de la organización.
Ocio. La categoría axiológica del ocio no parece relevante en el ambiente laboral, sin embargo, teniendo en cuenta que el salario es el intercambio de las horas de ocio por dinero, hace que la relación cobre importancia, por cuanto la necesidad de satisfacer el ocio no desaparece y requiere ser atendida. El exceso de trabajo tiende a generar en los colaboradores depresión, entornos hostiles, contrariedad, decepción y resentimiento, con lo cual se disminuye su productividad; en Fincomercio, este fenómeno genera despreocupación por la importancia de su labor, falta de planificación y ausencia de lugares de descanso.

Creación. Al igual que el entendimiento, la creación es una necesidad potencial en el ámbito laboral, donde las capacidades para innovar, crear, mostrar talentos, implican satisfactores de tipo sinérgicos y sentimientos de bienestar y de retribución a la sociedad. No obstante, en un ambiente en donde la creación y la innovación se vean limitadas por el estilo de liderazgo, se pueden generar múltiples insatisfacciones a los colaboradores. En el caso de estudio se señalaron el exceso de trabajo, las limitaciones de funciones y la desvalorización sobre ideas de mejoras o innovaciones como factores de desmotivación.

Identidad. La categoría axiológica de la identidad es una de las necesidades más humanas, es característica de la evolución del hombre en sociedad, brindándole sentido de pertenencia y seguridad. En el ámbito laboral, la identidad como organización tiende a prevalecer sobre la identidad individual, siendo en algunos casos generadora de insatisfactores como la dependencia, la incertidumbre, la negación y la divergencia. En Fincomercio, la inseguridad, la desigualdad, el aislamiento y la falta de integración son factores que afectan la identidad individual de sus colaboradores.

Libertad. Es el oxígeno a la vida y una categoría axiológica determinante para los individuos y las comunidades, permitiendo su nor- 
mal desarrollo. En términos laborales, la libertad tiende a verse limitada por las condiciones de la organización, los limites inherentes al cargo y las condiciones del entorno, tanto al interior de la empresa como en su nicho de mercado, generando en los colaboradores sentimientos de opresión, coacción, sumisión, injusticia, estereotipos, prejuicios, siendo posibles la manipulación, la polarización, la corrupción. Los prejuicios y la sumisión son factores que afectan el entorno laboral de los colaboradores de Fincomercio.

\section{Matriz del Bien Común como satisfactor sinérgico para Fincomercio}

Luego de la aplicación de la matriz de desarrollo a escala humana dentro de la organización, se identificó que las necesidades más susceptibles dentro de la misma están asociadas a las categorías subsistencia, protección y participación. Es im- portante aclarar que, la matriz no es normativa $y$ se construye de acuerdo a la experiencia que se tiene en su aplicación, permitiendo un diálogo participativo para su determinación. En este contexto, se propone realizar el balance del bien común, contemplando temas relacionados con los puestos de trabajo (asociado a la categoría de subsistencia, en la cual se muestra estrés, horarios laborales), ingreso (asociado a la protección) y participación (asociado a la misma categoría en la matriz de necesidades y satisfactores), por lo cual funciona como satisfactor sinérgico para que la organización evalúe su actuar frente a estos temas y reflexionar sobre qué acciones se puede tomar para mejorarlos; adicional dicha matriz contempla otros puntos relacionados con proveedores, clientes, medio ambiente, valorando el impacto externo que tiene la misma. En la creación del balance del bien común se obtuvieron los siguientes resultados:

\section{Tabla 2. Matriz del Bien Común Fincomercio. Año 2019}

\section{Calculadora del Bien Conún- Versión 5.02 MATRIZ DEL. BIEN COMÚN}

Emoresa: Fincomercio Ltda.: Ar̃o del balance:: 2019

\begin{tabular}{|c|c|c|c|c|}
\hline $\begin{array}{r}\text { Valars : } \\
\text { Chupos daintarts } \mathbf{F}\end{array}$ & Dgnidad humana & Soldaridad y Jutcla & sotenlbill fad med oamblental & $\begin{array}{c}\text { Tranaparancla y partcipacion } \\
\text { democratea }\end{array}$ \\
\hline \multirow[t]{2}{*}{ A:Provedoras } & $\begin{array}{l}\text { At: Digridad hurrarna en las } \\
\text { eaders de sumiristra }\end{array}$ & $\begin{array}{l}\text { A2: Justicia y soliderided an la } \\
\text { caderna da sumiristio }\end{array}$ & $\begin{array}{l}\text { A3: Sosteribildud mofosmbiartal an la } \\
\text { cudens de sumiristro }\end{array}$ & 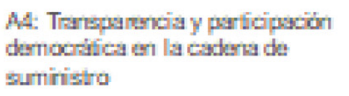 \\
\hline & 99 de 99 & 99 du 99 & 89 de 98 & 99 de 99 \\
\hline \multirow{2}{*}{$\begin{array}{l}\text { B: Proplatarios y } \\
\text { Proves dore: } \\
\text { finenclaros }\end{array}$} & 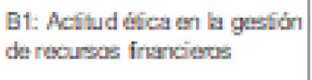 & $\begin{array}{l}\text { E2: Acifud solidaria an la } \\
\text { gestón da reoursos finarvious }\end{array}$ & 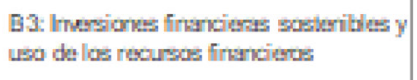 & $\begin{array}{l}\text { B4: Propiedad y participsoión } \\
\text { demoorified }\end{array}$ \\
\hline & 22 da 25 & 22 de 25 & $20 \mathrm{da} 25$ & $22 d u 25$ \\
\hline \multirow[t]{2}{*}{ C. Trabajadoras } & $\begin{array}{l}\text { C1: Digridad hurrarna eri el } \\
\text { puesta de tratueja }\end{array}$ & $\begin{array}{l}\text { C2: Famrididad de las ooritratos } \\
\text { de tratuja }\end{array}$ & 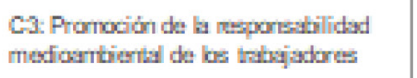 & 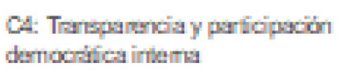 \\
\hline & $22 \mathrm{de} 25$ & 22 de 25 & 22 de 25 & 22 de 25 \\
\hline \multirow[t]{2}{*}{$\begin{array}{l}\text { D. cantes y otras } \\
\text { empreses }\end{array}$} & $\begin{array}{l}\text { Di: Actitud difica con las } \\
\text { dierites }\end{array}$ & 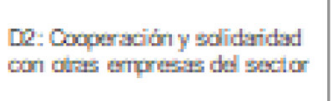 & 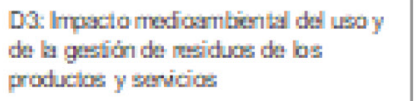 & 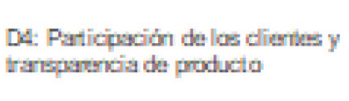 \\
\hline & 44 de 49 & 44 de 49 & 44 de 49 & 44 de 49 \\
\hline \multirow[t]{2}{*}{ E: Enterino exclal } & $\begin{array}{l}\text { E1: Proposista e impacio } \\
\text { pasifino de los productas y } \\
\text { senicios }\end{array}$ & $\begin{array}{l}\text { Q2: Contribución als } \\
\text { comuridad }\end{array}$ & $\begin{array}{l}\text { E3: Ruducción dal impucia } \\
\text { mesfosertiortal }\end{array}$ & 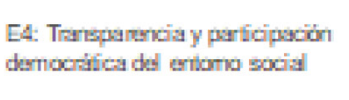 \\
\hline & 44 de 49 & 44 de 49 & 44 de 49 & 59 de 66 \\
\hline
\end{tabular}

Fuente: Elaboración propia con base en el ejercicio metodológico propuesto.
Nala esta ria es un centilicadg

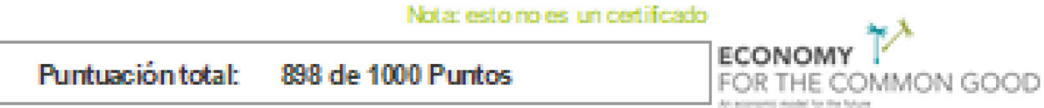

ECONOMY FOR THE COMMON GOOD

Transarencla y partcipaclon

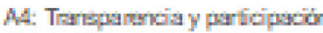
demoorifica en la caderta de 
El objetivo de la valoración, a través de la matriz presentada, es establecer el impacto de la actividad de una organización en el Bien Común. En el proceso de valoración, se analiza la actividad de la organización de acuerdo a una escala de valores; no se trata de una medición, sino de la aplicación de una puntuación otorgada a las actividades concretas de la organización y el análisis del efecto de las mismas en los diferentes grupos de interés. En el caso de Fincomercio, de un máximo de 1.000 puntos se obtuvo una calificación de 898 puntos, catalogada como una organización ejemplar, dentro del rango de puntuación establecido por el Equipo de Redactores del Balance del Bien Común. Esto significa que, en ella se cumple de manera óptima cada uno de los puntos evaluados por la matriz (alrededor de un 90\%), debido al manejo de buenas relaciones con sus proveedores, el respeto por la dignidad humana de los mismos, la relación contractual y demás requisitos exigidos por la ley en temas de pagos.

Desde la relación con los propietarios y proveedores financieros, se presenta una actitud ética hacia el manejo de los recursos financieros, pues muestra sus balances de manera anual, exponiendo cómo se lleva a cabo el manejo de los recursos y las decisiones de la compañía se toman de manera colectiva. En cuanto al tema de los colaboradores, se respetan los derechos del trabajador, existe la dignidad en los puestos de trabajo, la formalidad en los contratos establecidos con las prestaciones de ley, al igual que la generación de conciencia para los mismos en temas ambientales, por ejemplo, se realizan jornadas de ahorro de impresión, existen campañas para ahorro de papel y canecas para reciclar papel.

Para el tema de clientes, en el caso de la cooperativa de asociados, existe una actitud ética con los mismos, ayudas en temas de subsidios escolares, becas de manutención para estudiantes que se destacan en sus estudios, algunos subsidios para temas de calamidad (temas relacionados con salud física), descuentos en actividades relacionadas con temas de recreación, convenios con otras entidades en temas de salud, deporte, viajes y participación en las asambleas anuales para la toma de decisiones de la Cooperativa. Finalmente, en el entorno social se preocupan por impactar de manera positiva los lugares donde se encuentran ubicadas sus oficinas y que los habitantes conozcan los servicios que ofrece la Cooperativa, fomentando temas relacionados con la inclusión financiera.

\section{Planificación y evaluación}

Es importante aclarar que el balance del Bien Común es independiente de los balances tradicionales expuestos por la compañía. Así, las personas encargadas de realizar este balance deben tener noción del mismo, ser conocedores de la metodología y al menos hacer parte de la Organización del Bien Común 7 . Se recomienda realizar el proceso de evaluación cada dos años, con el fin de efectuar planes de mejora y fortalecer a la compañía en el tema del fomento del bien común. Se propondrá a la Cooperativa hacer actualizaciones cada dos años, por medio de consultoría, o mostrando los beneficios de hacer parte de la Organización.

En términos de desarrollo a escala humana, Max-Neef propone que para la política en América Latina, debe existir una generación y una asignación de recursos destinados a fortalecer las organizaciones locales que operan con racionalidad contra hegemónica, es decir, de manera solidaria, sinérgica y participativa, así como a incrementar

\footnotetext{
${ }^{7}$ Las empresas que han realizado la aplicación del balance del bien común son reconocidas como promotoras del bien común a nivel internacional en temas de contratación ética, comercio interno y externo justo, fomento del consumo ético y ejercicio de prácticas ambientales responsables. También han sido reconocidas por la Organización de las Naciones Unidas (ONU), por sus aportes a los objetivos de desarrollo sostenible (ODS). Algunas de estas compañías son Mondragón (Cooperativa del País Vasco, se considera la más grande del mundo), Sekem (Cooperativa egipcia de comercio justo), Grammen (Banco Indio de microcréditos y economía social), entre otras.
} 
la auto dependencia de estas organizaciones, promoviendo una transformación estructural y un cambio de lo micro a lo macro.

La aplicación de la metodología de Felber genera efectos macroeconómicos bastante importantes, debido a que para él, estas organizaciones o micro organizaciones son generadoras de trabajo, el cual constituye mucho más que un factor de producción pues "propicia la creatividad, moviliza energías sociales, preserva la identidad de la comunidad, despliega solidaridad, y utiliza la experiencia organizacional y el saber popular para satisfacer necesidades individuales y colectivas" (Max-Neef, Elizalde y Martín, 1986, p. 107). En términos de Max-Neef, existen seis elementos que son importantes dentro de la organización como se muestra en la siguiente figura:

Figura 1. Más allá de los recursos económicos.

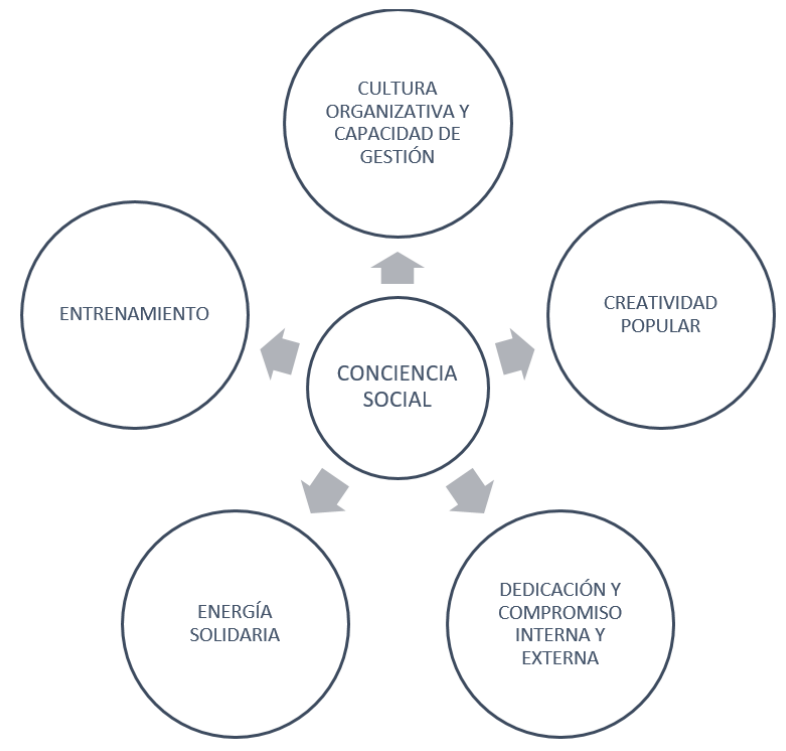

Los elementos anteriormente expuestos, se constituyen en satisfactores sinérgicos que fortalecen a la organización en temas de productividad y competitividad, y deben hacer parte de los procesos de mejora por parte de la organización, pues "potencian un desarrollo que va más allá de una noción convencional de acumulación, debido a que se basa lo práctico, a partir de la propia comunidad" (Max-Neef, Elizalde y Martín, 1986, p. 109)
El costo que debe asumir la empresa para convertirse en miembro activo de la Organización del Bien Común, se calcula de acuerdo al número de trabajadores que, para el caso de Fincomercio, son 400 empleados. De esta manera, el costo de vinculación está en un valor de 450 euros anuales (alrededor de $\$ 1.575 .000$ pesos colombianos). De esta manera, los costos asumidos por la compañía son los siguientes: inscripción a la Organización del Bien Común (\$1.575.000), taller de desarrollo a escala humana (\$1.200.000), consultor $(\$ 3.800 .000)$; para un total de inversión de $\$ 6.575 .000$ pesos.

Finalmente, es importante destacar que una empresa puede potencializar su plan estratégico mediante la incorporación de modelos económicos alternativos, encaminados a mejorar la productividad y la competitividad, más allá de un desarrollo tradicional. Como lo propone Max-neef, es preciso tener en cuenta el componente humano como el factor de trabajo más importante dentro de las mismas; y desde la economía del bien común se hace una propuesta más allá de lo convencional -responsabilidad social-, permitiendo una visión holística que reúne a todos los agentes de la sociedad y busca generar una maximización de los beneficios de los miembros de la organización, no sólo en términos de crecimiento sino también en términos de desarrollo humano, social, político y ambiental.

Para el caso de Fincomercio, se evidenció que es una Cooperativa que cumple a cabalidad con los temas establecidos en el fomento del bien común, y con los satisfactores identificados en la misma, se puede empezar a trabajar en la solución de temas relacionados con el estrés laboral, trabajo en equipo, limitaciones presentes en el desarrollo de las actividades laborales. Lo anterior deberá tener por objetivo fortalecer el capital humano de la Cooperativa y potencializar el impacto social organizacional y social de la misma. 


\section{Conclusiones}

La identificación de las necesidades y satisfactores de los colaboradores de una organización, genera un ambiente laboral diferente, debido a que se busca mejorar la calidad de vida y el bienestar de todas y todos. Lo anterior, trae beneficios para muchas empresas, pues con ello se da un aumento y potencialización de las capacidades de sus trabajadores, logrando que los empleados se sienten motivados y puedan ser más productivos en sus labores diarias.

Cuando se mejoran las prácticas empresariales, y se busca la forma de generar un desarrollo sostenible dentro y fuera de la organización, se consolida a la empresa dentro del mercado y se puede establecer como una empresa promotora del Bien Común en Colombia. Esta apuesta crea un valor agregado, al promover prácticas responsables que contribuyen al respeto y la práctica de valores universales. Además, al diseñar estímulos e incentivos a los sectores industriales y de presentación de servicios en el ámbito empresarial, tales como exenciones de impuestos, reconocimientos empresariales, distinciones, entre otros; al incorporar dichos modelos alternativos en sus planes estratégicos, se permitirá a las empresas evaluar sus desempeños más allá de indicadores financieros.

Las actividades de la organización, y su impacto en los grupos de interés, se ven reflejados ampliamente en las matrices expuestas en este documento, y a través de los niveles de aportación al Bien Común y el Desarrollo a Escala Humana; la valoración se realiza teniendo en cuenta los niveles de aportación al mismo (punto de partida, primeros pasos, avanzado, experimentado y ejemplar). Para este fin, debe utilizarse toda la información e indicadores disponibles como balances, formas de contratación, entrevistas, experiencias, entre otros. Los talleres propuestos en el desarrollo de las metodologías, permite tomar información más cercana a los colaboradores y mejores deci- siones dentro de la empresa. Esto se logra a través del fortalecimiento de la relación entre directivos y colaboradores, y el área de gestión humana es la encargada de hacer esta integración.

\section{Referencias bibliográficas}

Alfaro, A. (2016). El mercadeo a escala humana, el comercio justo y nuevos paradigmas de desarrollo. Obtenido de http://upagu.edu. pe/es/wp-content/uploads/2016/10/MERCADEO-A-ESCALA-HUMANA-Y-NUEVOS-PARADIGMAS-DE-DESARROLLO. pdf

Americaeconomía.com. (13 de Agosto de 2018). Mba \& educación ejecutiva. Obtenido de https://mba.americaeconomia.com/articulos/notas/desarrollar-desafios-junto-loscolaboradores-reduce-el-conformismo-laboral

Comisión Económica para América Latina . (19 de Agosto de 2019). CEPAL. Obtenido de https://www.cepal.org/es/areas-de-trabajo/ desarrollo-economico

Delfín, F., \& María, A. (2016). Importancia y análisis del desarrollo empresarial. Revista científica Pensamiento y gestión , 186-202.

Economic Analysis \& Policy Division. (2018). Situación y Perspectivas de la economía mundial 2018. Obtenido de https://www. un.org/development/desa/dpad/publication/situacion-y-perspectivas-de-la-economia-mundial-2018-resumen/

Equipo de redactores de la Matriz EBC. (2017). Manual del Balance del bien común 5.0. Balance completo.

Felber, C. (2012). Economía del Bien común. Viena: Paul Zsolnay Verlag. 
Fincomercio. Cooperativa de ahorro y crédito. (1 de Mayo de 2019). Obtenido de https:// www.fincomercio.com/corporativo/historia/

Guimón,P.(15deJuniode2015).Elpaís.Obtenidode https:/elpais.com/economia/2015/06/15/ actualidad/1434384197_133454.html

Hernández Sampieri, R., Fernández Collado, C., \& Baptista Lucio, P. (2014). Metodología de la Investigación. México, D.F.: Mc Graw Hill.

López, L. (30 de Mayo de 2013). Escuela de Organización Industrial. Obtenido de eoi.es/ blogs/mintecmad/el-crecimiento-economico-bienestar-para-la-sociedad/

Los hombres de ADE. (31 de Mayo de 2006). Obtenido de https://harrisseramado.wordpress. com/2006/05/31/necesidades-axiologicas/

Martínez, E. (12 de Diciembre de 2010). Alcaldía Mayor de Bogotá . Obtenido de http://observatorio.desarrolloeconomico.gov.co/ din $\%$ C3\% A 1 mica-econ $\%$ C3\%B 3 mica$\mathrm{y}-\mathrm{distribuci} \% \mathrm{C} 3 \% \mathrm{~B} 3 \mathrm{n} / \mathrm{des}$ arrolloecon $\%$ C $3 \%$ B 3 mico-vs-crecimientoecon $\% \mathrm{C} 3 \% \mathrm{~B} 3 \mathrm{mico}$
Max-neef, M., Elizalde, A., \& Martín, H. (1986). Desarrollo a Escala humana. Conceptos, aplicaciones y algunas reflexiones. Montevideo: Editorial Nordan-Comunidad.

Organización mundial de la salud. (1 de Mayo de 2019). Obtenido de https://www.who.int/ occupational_health/publications/stress/es/

Pereira, H. (2011). Centro de Gestión del Conocimiento. Obtenido de http://www.cegesti. org/exitoempresarial/publicaciones/publicacion_135_310111_es.pdf

Solo consultores en cambio . (Marzo de 2015). Factorhuma.org. Obtenido de https://factorhuma.org/attachments_secure/article/11575/ annex_Nuria_Povill.pdf

Weissman, I. (21 de Enero de 2019). Redacción. Obtenido de https://www.redaccion.com. ar/desigualdad-en-aumento-la-brecha-entre-ricos-y-pobres-en-el-mundo-sigue-creciendo/ 